\title{
Postdischarge Surveillance for Surgical Site Infection: The Continuing Challenge
}

\author{
Petra Gastmeier, MD
}

Surgical site infection (SSI) remains one of the most important problems in infection control. There is no doubt that SSIs substantially contribute to prolongation of hospital stay and increase costs. ${ }^{1-3}$ However, it is debatable to what extent they contribute to attributable mortality. Kirkland et al. ${ }^{1}$ found that the likelihood of death for patients with SSI is twice that for patients without SSI. A recent French study found that $38 \%$ of deaths involving patients with SSI were due to the infection, for an attributable mortality of $0.9 \%{ }^{4}$ In a British study published in 2005, only patients with SSI following hip prosthesis had a mortality rate that was significantly higher than that for patients without SSI (odds ratio, 1.8), ${ }^{3}$ but Whitehouse et al. ${ }^{5}$ did not find this difference in a study involving patients who underwent orthopedic surgery.

This issue of the journal includes a number of articles on SSI and postdischarge surveillance (PDS). The study by Herwaldt et al. ${ }^{6}$ analyzed data from a large, randomized, controlled study $\mathrm{y}^{7}$ that investigated the impact of intranasal mupirocin use for the prevention of postoperative Staphylococcus aureus infection and described the influence of nosocomial infections on mortality, prolongation of hospital stay, and other outcomes. The strengths of the study are the large number of patients involved ( $n=3,864$ ), the diversity of surgical specialities investigated (general surgery, neurosurgery, and cardiothoracic surgery), the large number of covariates considered (age, Karnofsky score, McCabe and Jackson classification, National Nosocomial Infection Surveillance system [NNIS] risk index, and number of comorbidities), a long mean postoperative follow-up period of 30 days, and the inclusion of time to infection in the analysis. ${ }^{6}$ A total of 438 patients acquired at least 1 nosocomial infection, and 316 of the 438 had SSIs. After accounting for covariates, postoperative nosocomial infection was not associated with a significantly increased risk of death (odds ratio, 1.14 [95\% confidence interval, 0.54-2.38]) but was associated with increased length of hospital stay (LOS) after surgery and increased costs. Herwaldt et al. ${ }^{6}$ demonstrate convincingly that, to accurately assess the effects of nosocomial infection, one must consider important covariates, and they confirm the important economic consequences of SSI.

Prevention of SSIs remains a major target of infection control personnel, and surveillance-in particular, participation in a surveillance network - has a substantial impact on decreasing SSI rates. ${ }^{8,9}$ However, over the past decade, there has been an increasing trend towards shorter LOS, which means the percentage of SSIs occurring after discharge is increasing in the majority of countries. Not considering differences in PDS of SSIs between hospitals may increase the risk of misleading conclusions when comparing data on SSI rates between hospitals.

Therefore, it is not surprising that colleagues from all over the world have investigated the methods and effects of PDS. In the past 5 years, investigators from Australia ${ }^{10,11}$ Brazil, $^{12}$ Estonia, ${ }^{13}$ France, ${ }^{14,15}$ Germany, ${ }^{16}$ The Netherlands, ${ }^{17}$ New Zealand, ${ }^{18}$ the United Kingdom, ${ }^{19-21}$ and the United States ${ }^{22-24}$ have demonstrated the potentially enormous impact of PDS on the rate of SSI in the populations they studied.

In this issue of the journal, 3 studies from Europe complement our growing knowledge about SSI detected through PDS. In a study from Italy, Prospero et al. ${ }^{25}$ investigated a relatively small group of patients $(n=264)$, using a traditional approach. Data were collected by 1 surgeon and 1 physician from the hospital hygiene service. If patients did not come for routine outpatient follow-up visits, they were contacted by telephone 30 days after the operation by the surgeon, who diagnosed SSI on the basis of questionnaire responses. Compliance with follow-up was 94\%. Approximately $60 \%$ of patients with SSI developed the infection after hospital discharge. Among NNIS operative procedures for which PDS detected SSIs, herniorrhaphy, mastectomy, other endocrine system surgery, and other integumentary system surgery were associated with a mean postoperative stay that was less than the mean time between the procedure and the onset of SSI. Therefore, Prospero et al. ${ }^{25}$ suggest changing from generalized PDS to operative procedure-specific PDS,

From the Institute for Medical Microbiology, Hannover Medical School, Hannover, Germany,

Received September 9, 2006; accepted September 9, 2006; electronically published November 27, 2006. Infect Control Hosp Epidemiol 2006; 27:1287-1290

C 2006 by The Society for Healthcare Epidemiology of America. All rights reserved. 0899-823X/2006/2712-0001\$15.00. 


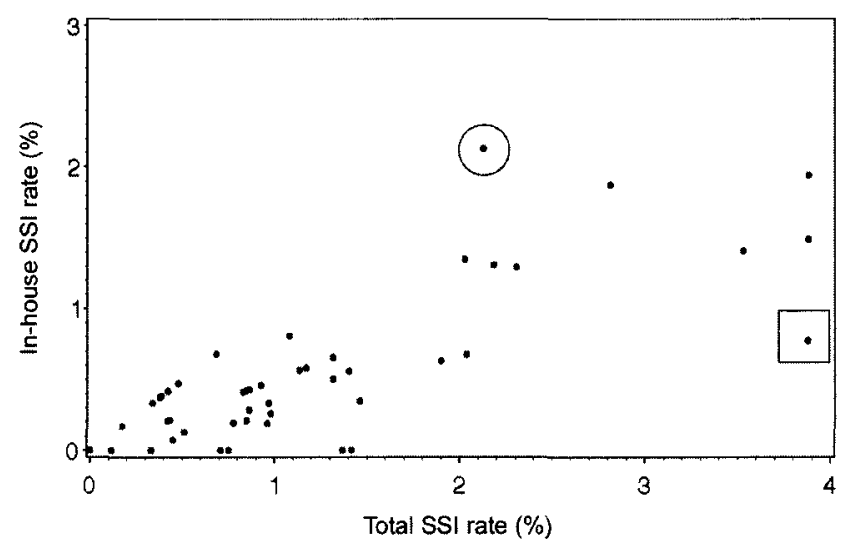

FIGURE. Distribution of 44 orthopedics departments in the German National Nosocomial Infection Surveillance System (Krankenhaus Infektions Surveillance System) according to the overall surgical site infection (SSI) rate (which includes postdischarge data) and the in-hospital SSI rate for knee replacement procedures. Data were recorded between January 2001 and June 2006. Each dot represents 1 orthopedics department. The circle indicates a department that only collects data on in-hospital SSIs. The square indicates the department that seems to have the largest overall SSI rate, but this is because of their activities to identify SSI after discharge; if only in-hospital data were included, this department would not be an outlier.

at least for procedures locally considered to have a high risk of SSI.

Instead of focusing on 1 hospital, Reilly et al. ${ }^{26}$ investigated the impact of PDS on SSI rates for selected surgical procedures in acute care hospitals throughout Scotland. Thirty-two of 46 acute care hospitals in Scotland contributed data on 21,710 surgeries categorized into 9 types of operative procedures. For each surgery, the local surveillance coordinators were asked to classify the PDS methods used by healthcare staff as none, passive, or active with or active without direct observation of the surgical site within 30 days after the procedure. PDS data were available for 12,885 operations (59\%); SSI PDS was passive in 2,793 operations (13\%) and active in $10,092(46 \%)$. Among the surgeries not associated with PDS, the rate of SSI was $2.6 \%$, which was significantly lower than the rate of SSI among surgeries associated with PDS (6.34\%). Among procedures for which PDS was performed, the rate of SSI was significantly higher for breast surgery, cesarean section, hip arthroplasty, and abdominal hysterectomy, compared with the rate among procedures for which PDS was not performed; no differences in SSI rates with or without PDS were found for surgery for fractured neck of the femur and knee arthroplasty. Therefore, Reilly et al. ${ }^{26}$ recommended a procedure-specific PDS approach with direct observation of patients after breast, cesarean and hysterectomy procedures, after which the LOS is often short. SSI surveillance at the time of readmission may be adequate to detect most SSIs after orthopedic surgery or vascular surgery, after which the LOS is longer.
A similar result was found in the study by Huotari and Lyytikainen, ${ }^{27}$ who concentrated on orthopedic surgery only and used data from 9 hospitals participating in the Finnish Hospital Infection Program. Because of the availability of a national identity code for each patient, it was possible to combine data from the hospital stay with data from followup out patient visits. Follow-up visits usually occurred 2 months and 1 year after hip or knee arthroplasty. In addition, 7 of 9 hospitals conducted PDS by means of a questionnaire, which was given to each patient at the time of discharge. The study included 11,812 procedures; 216 SSIs $(56 \%)$ were detected after discharge. Most (70\%) of the SSIs detected on readmission were severe (ie, deep incisional and organ/space infections), whereas most ( $86 \%$ ) of the SSIs detected during follow-up visits or by means of the PDS questionnaire were superficial. Thus, only a minority of deep incisional or organ/ space infections would be missed in patients for whom questionnaire-based PDS is not performed.

Taken together, these 3 informative studies may stimulate the reader to think about possible solutions to the challenge of designing surveillance methods for detection of SSI after discharge. These studies revealed that a high percentage of SSIs were detected after discharge, and if these findings are ignored, it is likely that many SSIs will go undetected and that the SSI rate will be substantially underestimated. However, direct examination of the wounds of all patients by trained professionals (regarded as the criterion standard for detection of SSI) is difficult and very expensive to perform. Therefore, a balance between the accuracy of PDS data and the cost-effectiveness of PDS activities has to be found for routine surveillance.

In some hospitals and countries, good technical solutions to this problem have been identified. Platt et al. ${ }^{28}$ and Sands et al. ${ }^{22}$ described automated surveillance methods based on pharmacy and financial claims data and reported that they are more sensitive for detection of postdischarge SSI, are less susceptible to interobserver variation, and provide more-precise estimates of infection rates. Michelson ${ }^{24}$ successfully tested whether text-based searching of standard dictated outpatient clinic notes could be used to detect postoperative infections that otherwise would not be detected by traditional hospital-based surveillance methods. However, these technical methods are currently only feasible in some hospitals or countries and therefore cannot be universally recommended for routine surveillance. Therefore, each national SSI surveillance system should identify the best method(s) for surveillance, on the basis of the capacity of the hospitals in the national health care system.

SSIs that become apparent after discharge also complicate comparisons within hospitals, between hospitals in the same country, and between hospitals in different countries. The Hospitals in Europe Link for Infection Control through Surveillance (HELICS) network was created to encourage the development of surveillance systems for detection of healthcare-acquired infection, to share expertise in surveillance for 
healthcare-acquired infection between countries, and to establish a European data set on healthcare-acquired infection by means of standardized definitions and methods to facilitate more meaningful comparison of rates between countries. Because of the activities of the HELICS network, we have a very good overview of SSI surveillance in Europe. Data from 16 regional surveillance systems in 13 countries were included in the 2004 report. $^{29}$ Centers for Disease Control and Prevention definitions were used to diagnose SSI, and the patient case-mix was considered, using International Classification of Diseases, Ninth Revision, procedure codes and the NNIS risk index. However, variations in observation periods also may affect the validity of comparing data from hospitals participating in the HELICS network. The median postoperative LOS varied between countries (eg, for coronary artery bypass surgery, the LOS was 6 days in Spain and 13 days in Lithuania; and for hip arthroplasty, the LOS was 6 days in Northern Ireland and 12 days in Austria). The detection of SSI after discharge in individual hospitals was entirely dependent on the intensity of their PDS, and there was considerable heterogeneity in data between countries. For instance, for SSIs following cesarean section, only inpatient data were considered in Austria and Spain, whereas 56\% of SSIs in France, $74 \%$ in Scotland, and $83 \%$ in The Netherlands were detected after discharge.

Participating hospitals in the HELICS network chose different approaches to address the difficulties in detecting and reporting SSIs that began after discharge. Hospitals in the Nosocomial Infection National Surveillance System in England decided to collect only information about SSIs detected during the hospital stay. ${ }^{30}$ The Prevention of Nosocomial Infections by Surveillance (Preventie Ziekenhuisinfecties door Surveillance; PREZIES) network in The Netherlands strongly recommends using 2 active surveillance methods, as described recently by Manniën et al. ${ }^{17}$ This is possible because in the Dutch healthcare system, almost every patient is seen again by the surgeon after hospital discharge. In the German healthcare system, there is strict separation between hospital and outpatient care, which is why only SSIs passively detected (ie, at readmission or at random) are included in the database. Therefore, the German Nosocomial Infection Surveillance System (Krankenhaus Infektions Surveillance System; KISS) specifies 2 SSI rates: the overall (ie, in-hospital plus outpatient) SSI rates for each type of surgical procedure in each surgical department are used for internal quality control, and the in-hospital SSI rate is used to avoid misleading conclusions when comparing SSI rates between departments (Figure 1). Similarly, the Centers for Disease Control and Prevention NNIS does not require PDS for SSIs. Some NNIS participants perform PDS and others do not, and the methods used for PDS vary by facility.

Because of the enormous differences in PDS between hospitals and countries, comparisons between hospitals and between countries should only be performed when it is possible to consider information about the extent and quality of PDS.
Hopefully, previous publications, together with the findings reported in this issue of the journal, will facilitate further refinement of SSI surveillance methods, including tailoring PDS in the most efficient, effective, cost-saving, and productive manner.

Address reprint requests to Petra Gastmeier, MD, Institute for Medical Microbiology, Hannover Medical School, Carl Neuberg-Str. 1, Hannover D 30 625, Germany (gastmeier.petra@mh-hannover.de).

\section{REFERENCES}

1. Kirkland K, Briggs J, Trivette S, Wilkinson W, Sexton D. The impact of surgical-site infections in the 1990s: attributable mortality, excess length of hospitalization, and extra costs. Infect Control Hosp Epidemiol 1999; 20:725-730.

2. Merle V, Germain J-M, Chamouni P, et al. Assessment of prolonged hospital stay attributable to surgical site infections using appropriateness evaluation protocol. Am J Infect Control 2000; 28:109-115.

3. Coello R, Charlett A, Wilson J, Ward V, Pearson A, Borriello P. Adverse impact of surgical site infections in English hospitals. J Hosp Infect 2005; 60:93-103.

4. Astagneau P, Rioux C, Golliot F, Bruker G. Morbidity and mortality associated with surgical site infections: results from the 1997-1999 INCISO surveillance. INCISO Network Study Group. J Hosp Infect 2001; 48: 267-274.

5. Whitehouse J, Friedman N, Kirkland K, Richardson W, Sexton D. The impact of surgical-site infections following orthopeadic surgery at a community hospital and a university hospital: adverse quality of life, excess length of stay, and extra costs. Infect Control Hosp Epidemiol 2002; 23: $183-189$.

6. Herwaldt L, Cullen J, Scholz D, et al. A prospective study of outcomes, healthcare utilization, and costs associated with postoperative nosocomial infections. Infect Control Hosp Epidemiol 2006; 27:1291-1298 (in this issue).

7. Perl T, Cullen J, Wenzel R, et al. Intranasal mupirocin to prevent postoperative Staphylococcus aureus infections. Mupirocin and the Risk of Staphylococcus aureus Study Team. N Engl J Med 2002; 346:1871-1877.

8. Geubbels E, Nagelkerke N, Mintjes-de Groot A, Vandenbrrroucke-Grauls C, Grobbee D, de Boer A. Reduced risk of surgical site infections through surveillance in a network. Int J Qual Health Care 2006; 18:127-133.

9. Gastmeier P, Geffers C, Brandt C, Zuschneid I, Sohr D, Schwab F, Behnke $M$, Daschner F, Rüden $\mathrm{H}$ : Effectiveness of a nationwide nosocomial infection surveillance system for reducing nosocomial infetions. J Hosp Infect 2006; 64:16-22.

10. Kent P, McDonald M, Harris O, Mason T, Spelman D. Post-discharge surgical wound infection surveillance in a provincial hospital: followuprates, validity of data and review of the literature. ANZ J Surg 2001; 71:583-589.

11. Noy D, Creedy D. Postdischarge surveillance of surgical site infections: a multimethod approach to data collection. Am J Infect Control 2002; 30:417-424.

12. Oliveira A, Carvalho D. Postdischarge surveillance: the impact on surgical site infection incidence in a Brazilian university hospital. Am I Infect Control 2004; 32:358-361.

13. Mitt P, Lang K, Peri A, Maimets M. Surgical-site infections following cesarean section in an Estonian university hospital: postdischarge surveillance and analysis of risk factors. Infect Control Hosp Epidemiol 2005; 26:449-454.

14. Thibon P, Parienti J-J, Borgey F, et al. Use of censored data to monitor surgical-site infections. Infect Control Hosp Epidemiol 2002; 23:361-363.

15. Barbut F, Carbonne B, Truchot F, et al. Surgical site infections after 
cesarean section: results of a five-year prospective surveillance. $J$ Gynecol Obstet Biol Reprod (Paris) 2004; 33:487-496.

16. Huenger $\mathrm{F}$, Schmachtenberg $\mathrm{A}$, Haefner $\mathrm{H}$, et al. Evaluation of postdischarge surveillance of surgical site infections after total hip and knee arthroplasty. Am I Infect Control 2005; 33:455-462.

17. Manniën J, Wille J, Snoeren R, van den Hof S. Impact of postdischarge surveillance on surgical site infection rates for several surgical procedures: results from the nosocomial surveillance network in The Netherlands. Infect Control Hosp Epidemiol 2006; 27:809-816.

18. Reid R, Simrock J, Chisholm L, Dobbs B, Frizelle F. Postdischarge clean wound infections: incidence underestimated and risk factors overestimated. ANZ J Surg 2002; 72:339-343.

19. Stockley J, Allen R, Thomlinson D, Constantine C. A district general hospital's method of post-operative infection surveillance including postdischarge follow-up, developed over a five-year period. I Hosp Infect 2001; 49:48-54.

20. Reilly J, Noone A, Clift A, et al. A study of telephone screening and direct observation of surgical wound infections after discharge from hospital. J Bone Joint Surg Br 2005; 87:997-999.

21. Johnson A, Young D, Reilly J: Caesarean section surgical site infection surveillance. J Hosp Infect 2006; 64:30-35.

22. Sands K, Yokoe D, Hooper D, et al. Detection of postoperative surgicalsite infections: comparison of health plan-based surveillance with hospital-based programs. Infect Control Hosp Epidemiol 2003; 24:741-743.
23. Sharma M, Berriel-Cass D, Baran JJ. Sternal surgical-site infection following coronary artery bypass graft: prevalence, microbiology, and complications during a 42-month period. Infect Control Hosp Epidemiol 2004; 25:468-471.

24. Michelson J. Improved detection of orthopaedic surgical site infections occuring in outpatients. Clin Orthop Relat Res 2005; 433:218-224.

25. Prospero E, Cavicchi A, Bacelli S, Barbadoro P, Tantucci L, D'Errico M. Surveillance for surgical site infection after hospital discharge: a surgical procedure-specific perspective. Infect Control Hosp Epidemiol 2006; 27: 1313-1317 (in this issue).

26. Reilly J, Allardice G, Bruce J, Hill R, McCoubrey J. Procedure-specific surgical site infection rates and postdischarge surveillance in Scotland. Infect Control Hosp Epidemiol 2006; 27:1318-1323 (in this issue).

27. Huotari $K$, Lyytikainen O. Impact of postdischarge surveillance on the rate of surgical site infection after orthopedic surgery. Infect Control Hosp Epidemiol 2006; 27:1324-1329 (in this issue).

28. Platt R, Yokoe D, Sands K. Automated methods for surveillance of surgical site infections. Emerg Infect Dis 2001; 7:212-216.

29. Hospitals in Europe Link for Infection Control through Surveillance. SSI Statistical Report 2004. Available at: http://helics.univ-lyon1.fr. Accessed March 2006.

30. Wilson J, Ward V, Coello R, Charlett A, Pearson A. A user evaluation of the Nosocomial Infection National Surveillance system: surgical site infection module. J Hosp Infect 2002; 52:114-121. 